Research Paper

\title{
Impaired Cognitive Executive Dysfunction in Adult Treated Hypertensives with a Confirmed Diagnosis of Poorly Controlled Blood Pressure
}

\author{
Christian Spinelli, Maria Fara De Caro, Gabriella Schirosi, Domenico Mezzapesa, Lorenzo De Benedittis, \\ Concetta Chiapparino, Gabriella Serio, Francesco Federico, Pietro Nazzaro
}

Department of Medical Basic Sciences, Neurosciences and Sense Organs, Division of Neurology-Stroke Unit, Hypertension, Medical School of Bari - University of Bari, Italy.

$\square$ Corresponding author: Pietro Nazzaro, MD. Department of Medical Basic Sciences, Neurosciences and Sense Organs, Division of Neurology - Stroke Unit, "FM. Puca", Unit of Cerebrovascular Prevention and Hypertension, University of Bari Medical School - Policlinico Consorziale, P.za G.Cesare,11 - 70124 BARI (Italy). Phone: ++39(080)5593659; Fax: ++39(080)5592253 E-mail: pietro.nazzaro@uniba.it.

() Ivyspring International Publisher. This is an open-access article distributed under the terms of the Creative Commons License (http://creativecommons.org/ licenses/by-nc-nd/3.0/). Reproduction is permitted for personal, noncommercial use, provided that the article is in whole, unmodified, and properly cited.

Received: 2013.II.I7; Accepted: 2014.02.27; Published: 2014.05.29

\begin{abstract}
Background. High blood pressure (BP) poses a major risk for cognitive decline. Aim of the study was to highlight the relationship between cognitive assessment scores and an effective therapeutic BP control.

Methods. By medical visit and ambulatory BP monitoring (ABPM), we studied 302 treated hypertensives, subdivided according to office/daytime BP values into 120 with good (GC) and 98 poor (PC) BP control, 40 with "white coat hypertension" (WCH) and 44 a "masked-hypertension" phenomenon (MH). Patients underwent neuropsychological assessment to evaluate global cognitive scores at the Mini Mental State Examination (MMSE) and Frontal Assessment Battery (FAB) and attention/executive functions (Delayed Recall, Digit Span Forwards, Digit Span Backwards, Selective Attention, Verbal Fluency, Stroop Test and Clock Drawing). Carotid intima-media thickness (IMT) served as the index of vascular damage.

Results. There were no differences among the groups in terms of gender, age, education, metabolic assessment, clinical history and hypertension treatment. GC presented lower office and ambulatory BP values and IMT. PC performed worse than GC on global executive and attention functions, especially executive functions. In PC, office systolic BP (SBP) was significantly associated to the MMSE and FAB scores and, in particular, to Verbal Fluency, Stroop Errors and Clock Drawing tests. Office diastolic BP (DBP) was associated to Selective attention, nocturnal SBP to Digit Span backwards and Verbal Fluency. Worse cognitive assessment scores were obtained in WCH than GC.
\end{abstract}

Conclusions. The findings showed that in adult treated hypertensives, a poor BP control, as both doctor's office and daytime scores, is associated to impaired global cognitive and especially executive/attention functions.

Key words: cognitive dysfunction, executive impairment, blood pressure control, ambulatory blood pressure monitoring.

\section{Introduction}

In recent years many studies have been focused on delineating the relationship between blood pressure (BP) and cognitive function, in view of the evidence that hypertension (HTN) is a risk factor for cognitive decline [1]. The findings are consistent for both vascular dementia and Alzheimer's disease [2] but, in particular, for the wider category of mild cognitive impairment [3]. The association between BP 
values and cognitive performances, in both adult hypertensives [4] and normotensives [5], is demonstrated by the progressive pathophysiological role of normal-to-high BP in worsening cognitive abilities. Nevertheless, the description of cognitive patterns associated with high $\mathrm{BP}$ is still controversial and largely debated. In fact, the cognitive tests employed and differences in patients' selection in the diverse studies may account for the heterogeneity of results [4-6].

According to a Cochrane review [6], the HYVET-COG study [7] and the SCOPE study [8] do not provide convincing evidence that pharmacological BP reduction can prevent the development of dementia in hypertensives without a prior diagnosis of cerebrovascular disease. Interestingly, only few studies have explored the different domains of cognitive functions that may be improved by effective BP control [9] but no study has compared cognitive scores with the hypertensive state findings during the medical visit and ambulatory BP monitoring (ABPM).

The aim of the study was to investigate the effect of BP control, both in the doctor's office and during daily activities, on cognitive functions in adults treated for hypertension. To assess cognitive function, we used specific tests that explore different cognitive domains.

\section{Methods}

Subjects. After obtaining approval for the study from the local Ethics Committee, between May 2009 and January 2012 we recruited 302 consecutive patients referred to the Cerebrovascular Prevention and Hypertension Clinic of the University of Bari Medical School.

The inclusion criteria were: a) age $\leq 69$ years, b) a history of primary hypertension, c) use of antihypertensive medications for at least six months, d) willingness to undergo a battery of neuropsychological tests and to give informed consent, e) Italian as native language, f) no previous history of psychiatric or neurological disorders including neuropsychological deficits, such as dementia, stroke or transient ischemic attack, g) no previous history of other cardiovascular diseases such as angina or myocardial ischemia, h) no history of episodes suggestive of cerebrovascular impairment, i) no atherothrombotic disease; 1) no history of diabetes and similar metabolic assessment. The "history of HTN" was defined as referred BP values, when available, and as estimated duration of the hypertensive condition in months. Patients in all four groups were taking antihypertensive treatment.

Study Protocol. Within one week of enrolment, patients presented at the clinic, between 8.00 and 9.00 a.m., after 12 hours fasting, for a routine medical visit, blood tests and electrocardiogram, to confirm the hypertensive state and to reduce the effect of anxiety on the BP measurement. Systolic (SBP) and diastolic (DBP) blood pressure and heart rate (HR) were considered as the mean of three measurements made using a standard sphygmomanometer (AND UM-101, A\&D Medical Ltd., Oxford, U.K), at 2 min intervals and at both arms, after the subject had been sitting comfortably for $10 \mathrm{~min}$. The patients underwent carotid ultrasonography, ambulatory blood pressure monitoring and cognitive assessment.

Carotid Ultrasonography. On the following day, carotid ultrasonography was performed, by echodoppler (Toshiba Aplio XV) with a multi-frequency 6.2-8.4 $\mathrm{MHz}$ pulse wave probe, to check in both common carotid arteries for signs of atherosclerosis and measure the intima-media thickness (IMT), which served as the index of preclinical vascular damage, according to the Mannheim IMT Consensus [10].

Ambulatory Blood Pressure Monitoring. Within four days, the patients underwent twenty-four-hour ambulatory blood pressure monitoring (ABPM) to evaluate the BP state during awake and sleeping hours (AND TM 2430, A\&D Instruments Ltd., Oxford, U.K), with the tailored cuff fastened on the non-dominant arm. Because all of them had already previously undergone the test, it is reasonable to assume that the technique did not arouse any particular distress. Measures were taken every $20^{\prime}$ throughout the test. Awake and sleeping time were based on diary card entries and interview. Eight patients underwent a repeat ABPM because of an insufficient number $(<80 \%)$ of measurements [11].

Following the ESC-ESH 2007 guidelines, based upon both the diagnosis of hypertension in the clinic (SBP/DBP> 140/90 $\mathrm{mmHg}$ ) and during awake hours by ABPM (SBP/DBP> 135/85 mmHg) [11,12], the patients were subdivided into four groups: a) 120 with good (GC) and b) 98 with poor BP control (PC), both with a confirmed diagnosis of a $\mathrm{BP}$ condition in the office and during awake hours, and, c) 40 hypertensives with a positive office-day ABPM BP change, corresponding to the "white-coat hypertension" phenomenon, better defined as "isolated clinic hypertension" (WCH) and d) 44 with a negative office-day $\mathrm{ABPM} B \mathrm{~B}$ change, corresponding to the "masked hypertension" phenomenon $(\mathrm{MH})$, both with an inconsistent diagnosis of a BP condition between office and daytime awake scores.

Cognitive Assessment. The next day, the patients underwent neuropsychological assessment by trained psychologists under the supervision of a clinical neuropsychologist. The test battery was designed to assess a full range of cognitive functions, standardized for the Italian population, and consisted of relia- 
ble and sensitive analytical tests for cognitive impairment, validated by the scientific literature and generally associated to a specific cerebral area. In particular, memory functions are under the control of the temporal lobes, whereas executive and attention functions are controlled by the prefrontal cerebral regions. The examination included measures of global cognitive and frontal functions, by the Mini Mental State Examination (MMSE) [13] and Frontal Assessment Battery (FAB) tests [14]. The MMSE consists of a brief 30-point questionnaire that is used to screen the general cognitive index, while the FAB consists of a rapid tool including six subtests that can be used to discriminate between a frontal dys-executive phenotype and Alzheimer's Type dementia. Memory was assessed by the Prose Memory Immediate and Delayed Recall [15] and Digit Span Forwards [16] tests, used to investigate verbal long-term memory and verbal short-term memory, respectively. The cognitive domains of attention and executive functions were measured by the Digit Span Backwards test [16] that examines verbal working memory. The Selective Attention Test was used to measure the ability to focus on specific stimuli excluding those that are not relevant to the properties of the target [16], and the Verbal Fluency test to analyze the speed of verbal production [17]. The Stroop Test investigates the ability, time $(\mathrm{T})$ and errors made $(\mathrm{E})$, when asked to inhibit and suppress automatic responses [18] and the Clock Drawing Test is used to assess visual-constructive abilities and mental planning [19], requiring the involvement of executive functions. The tests were not limited by a ceiling or floor effect and were, therefore, sensitive to subtle changes in cognitive performance in the range of $\mathrm{BP}$ values in all patients. Raw scores were adjusted for age and education. The neuropsychological assessment lasted about $45^{\prime}$.

Statistical Analysis. The findings were analyzed by the SPSS 18.0 statistical package assuming a $p<0.05$ limit of significance. Data are expressed as mean \pm standard deviation. The chi-squared test was used to explore differences between two groups on gender. Between-group analyses of BP, metabolic and neuropsychological variables were operated by independent $\mathrm{t}$-test in the patients with a confirmed (GC vs PC) or unconfirmed office high $\mathrm{BP}$ condition ( $\mathrm{PC}$ vs WCH and GC vs MH) by ABPM. The Mann-Whitney U-test was applied for not normally distributed data. To highlight further peculiarities, ANOVA, followed by Bonferroni post hoc test $(\mathrm{p}<0.05)$ were also performed. Partial Pearson test, adjusted for IMT, blood glucose, LDL and HDL cholesterol, triglycerides, waist circumference and history of hypertension, as potential confounders, was used to explore the relationships between neuropsychological variables and BP values.

\section{Results}

To examine the results in patients with a confirmed diagnosis of a BP condition, both in the doctor's office and during daily hours, we compared GC to PC. By contrast, to evaluate the results in patients with inconsistent hypertensive scores, we compared $\mathrm{WCH}$ to PC, who showed higher office BP values, and $\mathrm{MH}$ to GC, who presented lower office BP values. There were no differences among the groups in terms of gender, level of education and estimated history of hypertension and referred first-diagnosed SBP/DBP values. Only the $\mathrm{WCH}$ were older and had received less education than the PC. As expected, during the medical visit, the PC group presented significantly higher SBP/DBP values than the GC. (Table 1a). There were no significant differences in the number of pills taken (GC 1.83 \pm 0.76 , PC $1.80 \pm 0.70$, WCH $1.85 \pm 0.71$ and $\mathrm{MH} 1.81 \pm 0.74$, p:n.s.), nor in the dosages and anti-hypertensive drugs, such as ACE-inhibitors, AT1-antagonists, calcium-entry blockers, beta-blockers, and diuretics, employed at the time of the study (data not shown). The metabolic assessment was also similar among the patients although the $\mathrm{WCH}$ group showed a lower BMI and abdominal circumference than the PC who, as expected, showed higher microalbuminuria and IMT than the GC (Table $1 b)$.

Daytime SBP/DBP was higher in the PC $(144 \pm 12 / 87 \pm 7 \mathrm{mmHg})$ than in the GC $(125 \pm 8 / 78 \pm 6$ mmHg; $p<0.001)$ while the WCH $(123 \pm 8 / 76 \pm 5$ $\mathrm{mmHg})$ and $\mathrm{MH}(141 \pm 7 / 87 \pm 5 \mathrm{mmHg})$ groups presented lower $(p<0.001)$ and higher $(p<0.001)$ values than the PC and GC groups, respectively. The PC group showed higher nocturnal SBP/DBP $(125 \pm 15 / 72 \pm 8 \mathrm{mmHg})$ than the GC group $(104 \pm 11 / 64 \pm 8 \mathrm{mmHg} ; \mathrm{p}<0.001)$, while the $\mathrm{WCH}$ $(107 \pm 14 / 64 \pm 9 \mathrm{mmHg})$ and $\mathrm{MH}(117 \pm 11 / 71 \pm 8 \mathrm{mmHg})$ showed lower $(\mathrm{p}<0.001)$ and higher $(\mathrm{p}<0.001)$ values than the PC and GC, respectively (Fig.1).

The nocturnal SBP decrease was lower in the PC $(13.65 \pm 7.99 \%)$ than in the GC $(16.40 \pm 7.59 \% ; p<0.05)$. The SBP change during the medical visit in the PC $(1.08 \pm 8.16 \%)$ was higher than in the GC $(-2.60 \pm 9.33 \%$; $\mathrm{p}<0.001)$ and the SBP office-daytime changes in the $\mathrm{WCH}(14.65 \pm 6.35 \%)$ and $\mathrm{MH}(-13.58 \pm 7.50 \%)$ were, as expected, higher $(\mathrm{p}<0.001)$ and lower $(\mathrm{p}<0.001)$ than in the PC $(-2.60 \pm 9.33 \%)$ and GC $(1.08 \pm 7.91 \%)$, respectively.

Interestingly, the GC and PC groups, who had a confirmed diagnosis of hypertension in the doctor's office and during awake hours, showed crucial differences in cognitive assessment scores. In fact, the PC performed significantly worse than the GC on global 
cognitive and frontal tests (MMSE: $27.9 \pm 2$ vs $28.5 \pm 1.6$; $\mathrm{p}<0.05$ and FAB: $15.4 \pm 2.2$ vs $16.2 \pm 1.7 ; \mathrm{p}<0.05)$, on attention function (Selective Attention Test: $46.5 \pm 7,1$ vs $48.8 \pm 5.6 ; \mathrm{p}<0.01$ ), on executive functions (Verbal Fluency Test: $28.7 \pm 10.7$ vs $32.4 \pm 9.9 ; \mathrm{p}<0.01$ and Stroop
Test -Time: $21.6 \pm 9.8$ vs $19.2 \pm 10.1 ; p<0.05$.). No further significant differences in cognitive tests emerged: Digit Span backwards ( $3.5 \pm 0.7$ vs $3.5 \pm 0.9$; n.s.), Stroop Test-Errors ( $0.4 \pm 1.8$ vs $0.5 \pm 1.8$; n.s.) and Clock Drawing Test (9.2 \pm 1.7 vs $8.8 \pm 2.3$; n.s.) (Fig.2).

Table I. Patients characteristics at enrollment

\begin{tabular}{|c|c|c|c|c|}
\hline & GC & PC & $\mathrm{WCH}$ & $\mathrm{MH}$ \\
\hline \multicolumn{5}{|l|}{$\mathbf{A}$} \\
\hline $\mathrm{M} / \mathrm{F}(\mathrm{n})$ & $53 / 67$ & $44 / 54$ & $18 / 22$ & $20 / 24$ \\
\hline Age (years) & $56.38 \pm 9.61$ & $56.70 \pm 11.02$ & $61.79 \pm 9.93^{\circ \circ}$ & $55.65 \pm 10.48$ \\
\hline Education (years) & $10.99 \pm 4.49$ & $11.30 \pm 4.10$ & $9.64 \pm 4.66^{\circ}$ & $11.65 \pm 3.97$ \\
\hline HTN history (months) & $14.57 \pm 33.91$ & $15.54 \pm 30.83$ & $26.67 \pm 39.54$ & $6.08 \pm 4.09$ \\
\hline SBP history (mmHg) & $161 \pm 14$ & $163 \pm 11$ & $161 \pm 10$ & $163 \pm 10$ \\
\hline DBP history (mmHg) & $97 \pm 6$ & $96 \pm 8$ & $96 \pm 6$ & $96 \pm 7$ \\
\hline SBP office (mmHg) & $123 \pm 9$ & $147 \pm 14^{* * *}$ & $145 \pm 10$ & $124 \pm 8$ \\
\hline DBP office (mmHg) & $80 \pm 4$ & $90 \pm 8^{* * *}$ & $89 \pm 7$ & $80 \pm 6$ \\
\hline HR office (bpm) & $71 \pm 7$ & $71 \pm 9$ & $70 \pm 8$ & $71 \pm 8$ \\
\hline \multicolumn{5}{|l|}{ B } \\
\hline $\operatorname{ABDCIRC}(\mathrm{cm})$ & $102.09 \pm 10.89$ & $102.67 \pm 10.01$ & $98.44 \pm 9.98^{\circ}$ & $101.05 \pm 10.51$ \\
\hline BMI (Kg/m2) & $28.79 \pm 4.73$ & $28.82 \pm 4.35$ & $27.25 \pm 3.40^{\circ}$ & $28.31 \pm 4.89$ \\
\hline C-TOT (mg/dl) & $128.18 \pm 36.07$ & $121.42 \pm 30.58$ & $132.89 \pm 49.54$ & $119.33 \pm 29.80$ \\
\hline C-LDL (mg/dl) & $202.86 \pm 43.97$ & $196.16 \pm 29.24$ & $211.35 \pm 49,29^{\circ}$ & $199.00 \pm 35.44$ \\
\hline C-HDL (mg/dl) & $51.84 \pm 11.25$ & $51.22 \pm 14.21$ & $52.78 \pm 11.25$ & $52.40 \pm 12.66$ \\
\hline TRIGL (mg/dl) & $118.66 \pm 63.98$ & $142.44 \pm 129.08$ & $109.22 \pm 49.34$ & $126.96 \pm 99.37$ \\
\hline GLIC (mg/dl) & $99.38 \pm 20.04$ & $104.83 \pm 30.23$ & $106.37 \pm 34.64$ & $94.40 \pm 7.29$ \\
\hline INSULIN $(\mu \mathrm{UI} / \mathrm{ml})$ & $11.48 \pm 7.09$ & $11.40 \pm 6.52$ & $9.12 \pm 6.12$ & $9.93 \pm 5.52$ \\
\hline CREAT (mg/dl) & $0.84 \pm 0.17$ & $0.87 \pm 0.24$ & $0.87 \pm 0.23$ & $0.86 \pm 0.18$ \\
\hline VFG (ml/min) & $100.64 \pm 26.26$ & $99.85 \pm 26.99$ & $87.51 \pm 31.22$ & $102.07 \pm 23.47$ \\
\hline MICROALB (mg/l) & $15.37 \pm 12.24$ & $39.99 \pm 77.60^{*}$ & $14.76 \pm 11.50$ & $24.42 \pm 39.56$ \\
\hline $\mathrm{IMT}(\mathrm{mm})$ & $0.84 \pm 0.21$ & $0.95 \pm 0.26^{* * *}$ & $0.88 \pm 0.20$ & $0.80 \pm 0.17$ \\
\hline
\end{tabular}

GC: treated hypertensives with a confirmed satisfactorily controlled blood pressure both in the office and during awake hours; PC: treated hypertensives with a confirmed insufficiently controlled blood pressure both in the office and during awake hours; WCH: treated hypertensives with an inconsistent hypertension ("isolate clinic hypertension/white-coat hypertension effect" with $\mathrm{BP}>140 / 90 \mathrm{mmHg}$ in the office but diurnal $\mathrm{BP}<135 / 85 \mathrm{mmHg}$; MH: treated hypertensives with an inconsistent hypertension ("masked hypertension effect") with BP<140/90 mmHg in the office but diurnal BP $>135 / 85 \mathrm{mmHg}\left({ }^{*}: \mathrm{p}<0.05 ;{ }^{* *}: \mathrm{p}<0.01 ;{ }^{* * *}: \mathrm{p}<0.001\right)$. The results in the patients with a confirmed diagnosis of high BP, both in the doctor's office and during daily hours, were analyzed (independent t-test) considering GC and PC patients $\left({ }^{*}: \mathrm{p}<0.05 ;{ }^{* *}: \mathrm{p}<0.01\right.$; ***: $\mathrm{p}<0.001$ ). According to the BP values during the medical visit, the results in patients with an inconsistent BP state in the doctor's office and during daily hours were analyzed (independent $\mathrm{t}$-test) in the $\mathrm{WCH}$ and PC groups, that both showed higher office BP values, and in the MH and GC groups, that both presented lower office BP values $\left({ }^{\circ}: \mathrm{p}<0.05 ;{ }^{\circ}: \mathrm{p}<0.01 ;{ }^{\circ 00}: \mathrm{p}<0.001\right)$.

General characteristics. M/F:sex distribution; Age (years); Education (years); HTN history (m): history of hypertension (months); SBP history: first-diagnosed systolic hypertensive values ( $\mathrm{mmHg}$ ); DBP history: first-diagnosed diastolic hypertensive values ( $\mathrm{mmHg}$ ); office: BP and HR values measured at the doctor's office.

Metabolic and vascular characteristics. ABDCIRC: abdominal circumference in cm, BMI: body mass index in kg/ $\mathrm{m}^{2}$; C-: blood cholesterol in mg/dl; TOT: total; LDL: low-density lipoprotein; HDL: high-density lipoprotein; TRIGL: triglycerides in mg/dl; GLIC: blood glucose in mg/dl; INSULIN: blood insulin in m $\mu$ /l; CREAT: creatinine in mg/dl; VFG: estimated glomerular filtration rate by MDRD formula; MICROALB: microalbuminuria as mg/l; IMT: intima-media thickness in mm.

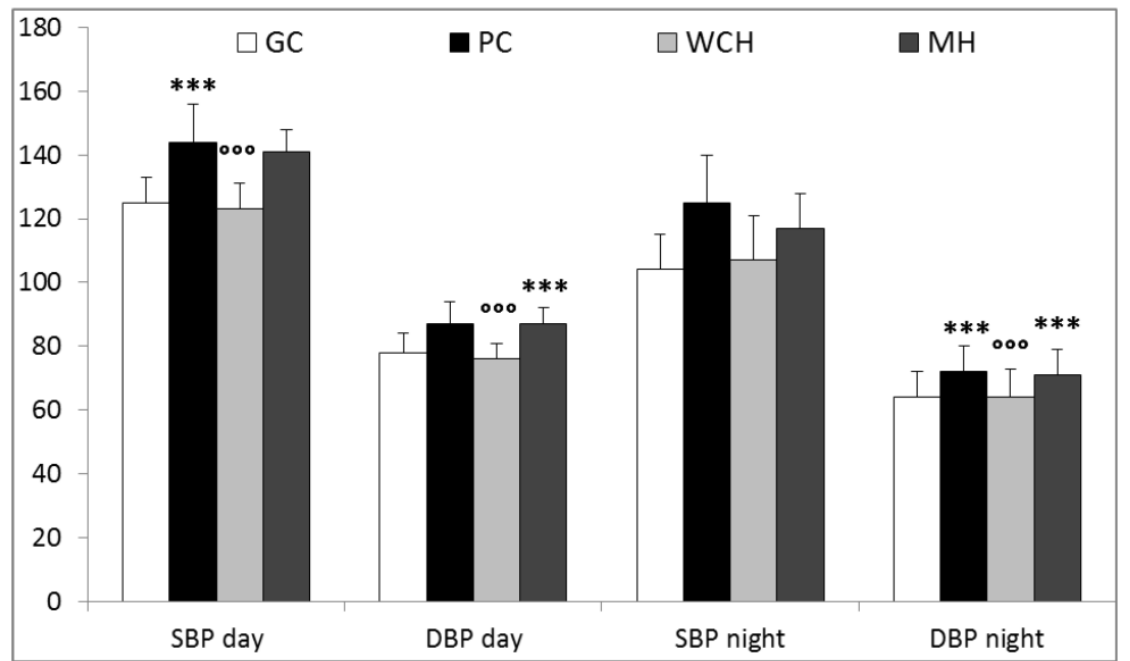

Figure I. Ambulatory blood pressure monitoring characteristics. GC: treated hypertensives with a confirmed satisfactorily controlled blood pressure both in the office and during awake hours; PC: treated hypertensives with a confirmed insufficiently controlled blood pressure both in the office and during awake hours; WCH: treated hypertensives with an inconsistent hypertension state ("isolated clinic hypertension/white-coat hypertension" effect with BP> I $40 / 90 \mathrm{mmHg}$ in the office but diurnal BP $<135 / 85$ $\mathrm{mmHg}$ during awake hours; $\mathrm{MH}$ : treated hypertensives with an inconsistent hypertension state ("masked hypertension" effect) with $\mathrm{BP}<140 / 90$ mmHg in the office but diurnal

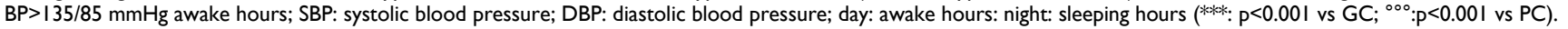




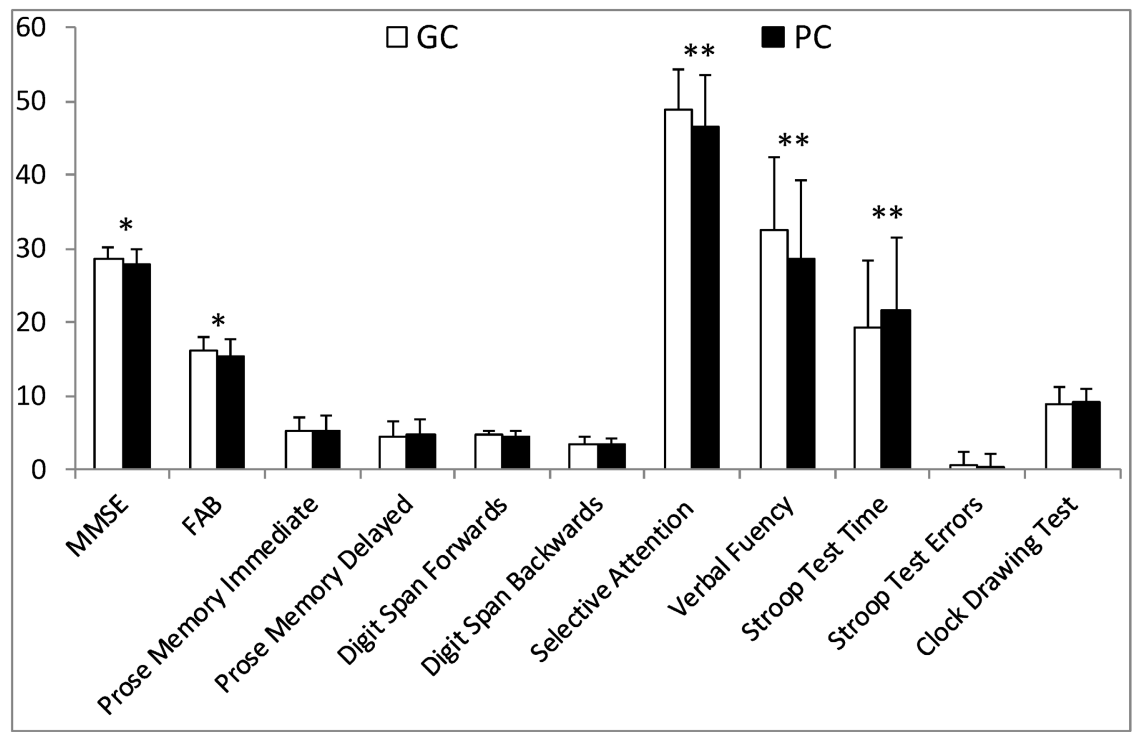

Figure 2. Neuropsychological assessment in treated hypertensives with a confirmed BP state. GC: treated hypertensives with a confirmed satisfactorily controlled blood pressure both in the office and during awake hours; PC: treated hypertensives with a confirmed insufficiently controlled blood pressure both in the office and during awake hours (*: $p<0.05, * *: p<0.01$ vs $W C H)$.

On the contrary, patients with a fluctuating and inconsistent BP condition exhibited very few differences at the cognitive evaluations. In fact, the $\mathrm{WCH}$ performed significantly worse than the PC only on the frontal test examined by the Digit Span Backwards (Table 2). No significant differences emerged between the $\mathrm{WCH}$ and $\mathrm{MH}$ on neuropsychological variables (Table 3).

All the patients completed the neuropsychological assessment and no participants were shown to have dementia or mild cognitive impairment. Although the major focus of the study was the office BP condition, in order to compare patients with a confirmed or no confirmed diagnosis of hypertension, we also performed one-way ANOVA, corrected by the Bonferroni test. This confirmed that the PC performed significantly worse than the GC on attention/executive tests. Interestingly, the $\mathrm{WCH}$ performed worse than the GC on the FAB (-1.356; $p=0.002)$, Digit Span backwards $(-0.468 ; p=0.016)$, Verbal Fluency $(-4.688 ; p=0.037)$ and Clock Drawing test $(-1.123 ; \mathrm{p}=0.028)$.

Pearson analysis, controlled for age, metabolic variables, history of HTN and first-diagnosed BP values as potential confounders, highlighted significant associations between cognitive test scores and BP values. The GC showed associations between nocturnal DBP and Digit Span forwards $(-0.210 ; p<0.05)$ and Digit Span backwards $(-0.201 ; \mathrm{p}<0.05)$ and between nocturnal SBP and Stroop test Errors (0.235; $\mathrm{p}<0.05)$. The PC demonstrated a significant association between office SBP and the MMSE (-0.304; $\mathrm{p}<0.01)$, FAB (-.0226; $\mathrm{p}<0.05)$, Verbal Fluency $(-0.220$; $\mathrm{p}<0.05)$, Stroop test Errors $(0.356 ; \mathrm{p}<0.001)$ and Clock Drawing test $(-0.372 ; p<0.001)$, between office DBP and Selective attention $(-0.210 ; \mathrm{p}<0.05)$ and between nocturnal SBP $(-0.211 ; \mathrm{p}<0.05)$ and Verbal Fluency $(0.210 ; p<0.05)$. Among the patients with no confirmed diagnosis of hypertension in the office and during awake hours, only the $\mathrm{WCH}$ showed a significant association between office DBP and Selective Attention $(-0.356$; $\mathrm{p}<0.05)$ whereas $\mathrm{MH}$ did not show any correlation. Interestingly, IMT was associated to frontal capacities, such as selective attention $(-0.525$; $\mathrm{p}<0.05$ ) only in the PC group.

Table 2. Cognitive assessment in $\mathrm{PC}$ and in $\mathrm{WCH}$ patients. Cognitive assessment, as scores adjusted for age and education, in treated hypertensives.

\begin{tabular}{lcc}
\hline & PC & WCH \\
\hline Global abilities & & \\
MMSE & $27.9 \pm 2$ & $27.9 \pm 2$ \\
FAB & $15.4 \pm 2.4$ & $14.9 \pm 2$ \\
Memory & & \\
Prose memory immediate & $5.2 \pm 2$ & $4.9 \pm 1.7$ \\
Prose memory delayed & $4.7 \pm 2.1$ & $4.6 \pm 1.9$ \\
Digit Span forwards & $4.5 \pm 0.8$ & $4.3 \pm 0.7$ \\
Attention and executive functions & & \\
Digit Span backwards & $3.5 \pm 0.9$ & $3.0 \pm 0.8 * * *$ \\
Selective attention & $46.5 \pm 7.1$ & $48.0 \pm 6$ \\
Verbal fluency & $28.7 \pm 10.7$ & $27.7 \pm 8.4$ \\
Stroop Test -Time & $21.4 \pm 9.9$ & $23.3 \pm 15.3$ \\
Stroop Test - Errors & $0.5 \pm 1.8$ & $0.3 \pm 0.8$ \\
Clock Drawing Test & $8.8 \pm 2.3$ & $8.1 \pm 3$ \\
\hline
\end{tabular}

PC: treated hypertensives with a confirmed insufficiently controlled blood pressure both in the office and during awake hours; $\mathrm{WCH}$ : treated hypertensives with an inconsistent hypertension state ("white-coat hypertension effect" with BP>140/90 $\mathrm{mmHg}$ in the office but diurnal $\mathrm{BP}<135 / 85 \mathrm{mmHg}$ during awake hours $\left(^{*}: \mathrm{p}<0.05\right.$; $\left.{ }^{* *}: p<0.01 ; * * *: p<0.001\right)$. 
Table 3. Cognitive assessment in GC and in $\mathrm{MH}$ patients. Cognitive assessment, as scores adjusted for age and education, in treated hypertensive patients.

\begin{tabular}{llc}
\hline \multicolumn{1}{l}{ GC } & MH \\
\hline Global abilities & & \\
MMSE & $28.5 \pm 1.6$ & $28.2 \pm 1.4$ \\
FAB & $16.2 \pm 1.7$ & $16.1 \pm 2$ \\
Memory & \\
Prose memory immediate & $5.1 \pm 1.9$ & $5.2 \pm 1.8$ \\
Prose memory delayed & $4.5 \pm 2$ & $4.5 \pm 1.7$ \\
Digit Span forwards & $4.6 \pm 0.7$ & $4.6 \pm 0.6$ \\
Attention and executive functions & & \\
Digit Span backwards & $3.5 \pm 0.7$ & $3.3 \pm 0.7$ \\
Selective attention & $48.8 \pm 5.6$ & $48.9 \pm 4.5$ \\
Verbal fluency & $32.4 \pm 9.9$ & $30.4 \pm 6$ \\
Stroop Test -Time & $19.4 \pm 10.1$ & $19.3 \pm 8.8$ \\
Stroop Test - Errors & $0.4 \pm 1.8$ & $0.2 \pm 0.9$ \\
Clock Drawing Test & $9.2 \pm 1.7$ & $8.8 \pm 2.4$ \\
\hline GC: treated hypertensives with a confirmed satisfactorily controlled blood pressure \\
both in the office and during awake hours; MH: treated hypertensives with an \\
inconsistent hypertension state ("masked hypertension effect") with $\mathrm{BP}<140 / 90$ \\
mmHg in the office but diurnal BP $>135 / 85$ mmHg during awake hours (*: $\mathrm{p}<0.05 ;$ \\
$* * *$ p $<0.01 ; * * *$ p $<0.001)$.
\end{tabular}

\section{Discussion}

In the present study, adult treated hypertensives with a similar history of hypertension, metabolic assessment and treatment were evaluated to probe the effect of BP control, measured both in the doctor's office and during daily activities, on global cognitive functions and more definite attention-executive abilities evaluated by trained neuropsychologists.

The findings highlighted that the GC group showed a more effective cognitive performance than the PC. In particular, GC had better performances on global and frontal cognitive indexes, selective attention, and verbal fluency and Stroop time tests. The results suggest that an efficient and consistent BP reduction might be associated to a protective/beneficial effect on the preservation of cognitive functions.

The PC patients exhibited both a greater preclinical vascular damage, in terms of a higher IMT and increased microalbuminuria and the most extensive and strongest association between multiple BP measurements and reduced performances at global and attention/executive cognitive tests.

The findings suggest that the diagnosis of a limited BP control should be extended outside the doctor's office and validated by ABPM, crucial likewise to diagnose isolated clinical or masked hypertension and to highlight the risk for functional and structural cerebro-cardiovascular damage [20]. $\mathrm{WCH}$, with similar office BP but lower awake BP values, showed a limited association with the cognitive assessments and worse cognitive scores than GC. The findings confirm that "white -coat" hypertension is not a harmless condition, and is associated to a greater emotional cardiovascular reactivity [21] and enhanced cardiovascular risk [22]. Therefore, it is reasonable to assume that treated hypertensives who exhibit a "white-coat phenomenon" may also feature more impaired cognitive performances [23, 24].

Antihypertensive medications seem to reduce the risk for both the development and progression of dementia [25] and to improve cognitive functions in hypertensives without dementia [26]. Nevertheless, no relationship between a pharmacological BP reduction and the onset of cognitive impairment was found in elderly and very old hypertensives without prior cerebrovascular disease [6]. Indeed, some authors have hypothesized the existence of an age-dependent relation between $\mathrm{BP}$ and cognitive function, which would limit the benefits of an efficient BP control only to middle-aged patients but not the elderly [27]. Our findings, focusing on similarly treated adult hypertensives, support the hypothesis that a poor cognitive function in old age may be the result of a long-term exposure to vascular risks, such as hypertension, spanning more than 2 to 3 decades [28].

The role of antihypertensive treatment has been widely investigated in the last years with the intent of highlighting the efficacy of specific pharmacological subclasses on hypertension-dependent cognitive impairment [26] and demonstrating an additional "neuro-protective" effect of some molecules [27]. Our findings, on the contrary, seem to suggest that regardless of the active agent employed, the treatment may have a true influence on cognitive functions only when the BP control is effectively achieved both inside and outside the doctor's office. The "neuro-protection" should be, therefore, ascribed more to the BP level reached than to any particular antihypertensive drug used.

The previous studies were focused largely on global neuropsychological functions and only few papers combined an evaluation of impairments of the prefrontal functions such as executive function and working memory [29-32]. In our study, PC patients showed lower performances not only in terms of global executive capacities but, in particular, of selective attention and verbal fluency, suggesting that high $\mathrm{BP}$ values may have an early negative effect on frontal functions.

The relationship between hypertension and cognitive decline, according to the most widely accepted hypothesis, is mediated by cerebral vascular damage such as white matter injuries, detectable as white matter hyperintensities [33, 34]. Indeed, the disruption of white matter tracts that connect the frontal lobes to other cortical and subcortical structures [35] may affect attention/executive functions and speed processing $[36,37]$.

Antihypertensive treatment has been demonstrated to restrain the evolution of white matter le- 
sions [38]. It is reasonable, therefore, to hypothesize that a protective effect on deep subcortical white matter pathways, exerted by a proper BP control, might reduce the influence of hypertension on cognition, by improving the cerebral blood flow. The strict relationship between vascular damage and cognitive decline has been proven also by the evidence that the carotid IMT may predict cognitive decline among individuals lacking any vascular or neurological disease [39]. Likewise, we found that the IMT was inversely associated to attention functions in the PC but not in the other patients.

Some authors have proposed an additional restorative effect of a BP decrease within normal values on cognitive functions, especially on the attention/executive domain [40] but this was not confirmed by others who, on the contrary, reported poorer cognitive functions in treated mild hypertensives with an effective office BP control [41]. The discrepancy may be ascribed to the different consequences of hypertension on the vasculature and on the cerebral blood flow at different ages. This might be attributed to a dysfunction of the cerebral blood flow caused by the thickness and rarefaction of the perforating arterioles. Indeed, adult hypertensives affected by the metabolic syndrome showed a structural microvascular skin rarefaction, strictly associated to an impaired cerebrovascular arterial dilatation [42]. As compared to normotensives, hypertensives present a progressive decrease in cerebral blood flow mainly to the hippocampus, anterior cingulate gyrus and prefrontal cortex, areas considered to be involved in memory, executive function and attention [43]. Accordingly, the IMT was higher in the PC group despite the similar treatment, age, metabolic assessment and estimated histories of hypertension.

In middle-aged patients, antihypertensive treatment shows a reparative effect on brain perfusion with a major improvement in attention and psychomotor speed [44]. Then, in later life, the failure of the cerebral blood flow regulation, due to established endothelial and parietal arterial damage, triggers a compensating counter-regulatory mechanism, i.e. a higher BP state to maintain an optimal cerebral blood flow.

Our findings strongly suggest that a strict control, including ambulatory blood pressure monitoring, of the efficacy of antihypertensive treatments should be exerted to restrain cerebrovascular impairment from the very early stages of hypertension also in adult patients.

We are aware that our study suffers from some limitations. First of all, although we estimated the histories of hypertension and BP values, we cannot exclude that the PC group might have been affected by a more severe hypertension with secondary detrimental consequences or, alternatively, they could have been exposed to a prolonged and/or misdiagnosed resistant hypertension.

We cannot identify a causal effect among the range of variables and, because of the cross-sectional nature of the study, the effect of the estimated history of hypertension and BP levels, as well as the restorative effects exerted by different antihypertensive agents on cognitive function, cannot be estimated. Moreover, we were unable to obtain imaging data for all subjects. Then, the pathophysiology underlying the association of hypertension and attention/executive neuropsychological impairment can only be speculative, based on the most widely accepted hypotheses [33, 34, 45].

To the best of our knowledge, the present study is one of the very few to determine the outcomes of BP values obtained in the office and during the daily activities, on cognitive functions in an adult population. Whereas most of the authors investigated mainly global cognitive functions in older patients [46, 47], the present study was performed by trained psychologists who applied a range of cognitive assessments through a specific and extensive neuropsychological tools. The ABPM and multiple cognitive tools administered by neuropsychologists made it possible to ensure an effective diagnosis of the BP condition and proper testing procedures.

In conclusion, adult treated hypertensives with an insufficient BP control might suffer from an accelerated deterioration of cognitive performances, mainly in the functions regulated by frontal-subcortical circuits. The findings also highlight the critical role of ABPM to obtain a more reliable diagnosis of hypertension, to recognize the individual $\mathrm{BP}$ load, the benefit of treatment and any office-daytime BP difference. Further studies are needed to explore the effect of BP control on both peculiar cognitive functions and potential mechanisms whereby hypertension is associated with neuropsychological impairments.

\section{Acknowledgment}

We are indebted to Prof. Margherita Fanelli, Dept. of Medicine, University of Bari, for the statistical assistance and to Mary Victoria Pragnell, B.A., for language revision of the manuscript.

\section{Abbreviations} toring

ABPM: 24h ambulatory blood pressure moniBP: blood pressure

DBP: diastolic blood pressure

ESC-ESC: European Society of Cardiology - Eu- 
ropean Society of Hypertension

FAB: Frontal Assessment Battery

GC: treated hypertensives with a good control of

hypertension

HR: heart rate

HTN: hypertension

IMT: carotid intima-media thickness

$\mathrm{MH}$ : treated hypertensives with masked-

hypertension phenomenon

MMSE: Mental State Examination

PC: poorly-controlled treated hypertensives

SBP: systolic blood pressure

WCH: treated hypertensives with white-coat (or isolated clinic) hypertension phenomenon

\section{Competing Interests}

The authors have declared that no competing interest exists.

\section{References}

1. Viswanathan A, Rocca WA, Tzourio C. Vascular risk factors and dementia. How to move forward? Neurology 2009; 72: 368-374.

2. Duron E, Hanon O. Vascular risk factors, cognitive decline, and dementia. Vasc Health Risk Manag. 2008; 4:363-381.

3. Cherbuin N, Reglade-Meslin C, Kumar R et al. Risk factors of transition from normal cognition to mild cognitive disorder: the PATH through Life Study. Dement Geriatr Cogn Disord 2009; 28:47-55.

4. Kilander L, Nyman H, Boberg M. et al. Hypertension is related to cognitive impairment: a 20-year follow-up of 999 men. Hypertension 1998; 31:780-786.

5. Knecht $\mathrm{S}$, Wersching $\mathrm{H}$, Lohmann $\mathrm{H}$ et al. High-normal blood pressure is associated with poor cognitive performance. Hypertension 2008; 51: 663-668.

6. McGuinness B, Todd S, Passmore P et al. Blood pressure lowering in patients without prior cerebrovascular disease for prevention of cognitive impairment and dementia. Cochrane Database Syst Rev 2009: 7:CD004034

7. Peters R, Beckett N, Forette F et al.for HYVET investigators. Incident dementia and blood pressure lowering in the Hypertension in the Very Elderly Trial cognitive function assessment (HYVET-COG): a double-blind, placebo controlled trial. The Lancet Neurology 2008; 7: 683-689.

8. Lithell H, Hansson L, Skoog I et al. for SCOPE Study Group. The study on cognition and prognosis in the elderly (SCOPE): principal results of a randomized double-blind intervention trial. Journal of Hypertension 2003; 21: 875-886

9. Birns J, Morris $\mathrm{R}$, Donaldson $\mathrm{N}$ et al. The effects of blood pressure reduction on cognitive function: a review of effects based on pooled data from clinical trials. J Hypertens 2006; 24:1907-1914.

10. Touboul PJ, Hennerici MG, Meairs S et al. Mannheim carotid intima-media thickness consensus (2004-2006). An update on behalf of the Advisory Board of the 3rd and 4th Watching the Risk Symposium, 13th and 15th European Stroke Conferences, Mannheim, Germany, 2004, and Brussels, Belgium, 2006. Cerebrovasc Dis 2007; 23:75-80.

11. Mancia G, De Backer G, Dominiczak A et al. for ESH-ESC Task Force on the Management of Arterial Hypertension. 2007 ESH-ESC Practice Guidelines for the Management of Arterial Hypertension: ESH-ESC Task Force on the Management of Arterial Hypertension. ESC-ESH 2007 Guidelines. J Hypertens. 2007; 25:1751-1762

12. Pickering TG, Eguchi K, Kario K. Masked hypertension: a review. Hypertens Res 2007; 30:479-488

13. Measso G, Cavarzeran F, Zappalà G et al. The Mini-Mental State Examination: normative study of a random sample of Italian population. Dev Neuropsychol 1993; 9: 77-85

14. Appollonio I, Leone $\mathrm{M}$, Isella $\mathrm{V}$ et al. The frontal Assesment Battery (FAB): normative values in an Italian population sample. Neurological Sciences 2005; 26:108-116.

15. Bianchi A, Dai Prà M. Twenty years after Spinnler and Tognoni: new instruments in the Italian neuropsychologist's toolbox. Neurological Sciences September 2008; 29: 209-217

16. Orsini A, Grossi D, Capitani E et al. Verbal and spatial immediate memory span: normative data from 1355 adults and 1112 children. Italian Journal of Neurological Sciences 1987; 8:539-548.

17. Carlesimo GA, Caltagirone C, Gainotti G. The Mental Deterioration Battery: normative data, diagnostic reliability and qualitative analyses of cognitive impairment. The Group for the Standardization of the Mental Deterioration Battery. Eur Neurol 1996; 36: 378-384
18. Caffarra P, Vezzadini G, Dieci F et al. Una Versione Abbreviata Del Test Di Stroop: Dati Normativi Nella Popolazione Italiana. Nuova Rivista Di Neurologia 2002; 12: 111-115

19. Mondini S, Mapelli D, et al. Esame Neuropsicologico Breve - una batteria di test per lo screening neuropsicologico. Milano, Italy: Raffaello Cortina Editore; 2003.

20. Pickering TG, White WB, Giles TD, et al. When and how to use self (home) and ambulatory blood pressure monitoring. J Am Soc Hypertens 2010; 4: 56-61.

21. Tanya MS, Thomas GP, Joseph ES et al. The impact of perceived hypertension status on anxiety and the white coat effect. Annals of Behavioral Medicine 2007; 34: 1-9.

22. Muldoon MF, Nazzaro P, Sutton-Tyrrell K et al. White coat hypertension and carotid artery atherosclerosis: a matching study. Arch Intern Med 2000; 160:1507-1512

23. Suemoto CK, Nitrini R, Grinberg LT et al. Atherosclerosis and dementia: a cross-sectional study with pathological analysis of the carotid arteries. Stroke. 2011; 42: 3614-5.

24. Wetherell JL, Reynolds CA, Gatz M, et al. Anxiety, cognitive performance, and cognitive decline in normal aging. J Gerontol B Psychol Sci Soc Sci. 2002; 57: 246-55.

25. Shah K, Qureshi SU, Johnson $\mathrm{M}$ et al. Does use of antihypertensive drugs affect the incidence or progression of dementia? A systematic review. Am J Geriatr Pharmacother 2009; 7: 250-261.

26. Shlyakhto E. Observational Study on Cognitive function And systolic blood pressure Reduction (OSCAR): preliminary analysis of 6-month data from > 10,000 patients and review of the literature. Curr Med Res Opin 2007; 23 (Suppl 5):S13-S18

27. Qiu C, Winblad B, Fratiglioni L. The age-dependent relation of blood pressure to cognitive function and dementia. Lancet Neurol 2005; 4:487-499.

28. Joosten H, van Eersel MEA, Gansevoort RT et al. Cardiovascular risk profile and cognitive function in young, middle-aged and elderly subjects. Stroke 2013; 44: 1543-1549

29. Gupta R, Solanki RK, Pathak V. Blood pressure is associated with cognitive impairment in young hypertensives. World J Biol Psychiatry 2008; 9: 43-50.

30. Raz N, Rodrigue KM, Acker JD. Hypertension and the brain: vulnerability of the prefrontal regions and executive functions. Behav Neurosci 2003; 117: 1169-1180.

31. Harrington F, Saxby BK, McKeith IG et al. Cognitive performance in hypertensive and normotensive older subjects. Hypertension 2000; 36:1079-1082.

32. Swan GE, Carmelli D, Larue A. Systolic blood pressure tracking over 25 to 30 years and cognitive performance in older adults. Stroke 1998; 29: 2334-2340.

33. Sierra C, De La Sierra A, Salamero M et al. Silent cerebral white matter lesions and cognitive function in middle-aged essential hypertensive patients. Am J Hypertens 2004; 17: 529-534.

34. Gons RA, de Laat KF, van Norden AG et al. Hypertension and cerebral diffusion tensor imaging in small vessel disease. Stroke 2010; 41: 2801-2806.

35. Cummings JL. Frontal-subcortical circuits and human behavior. Arch Neurol 1993;50: 873-880.

36. Prins ND, van Dijk EJ, den Heijer T et al. Cerebral small-vessel disease and decline in information processing speed, executive function and memory. Brain 2005; 128: 2034-2041.

37. O'Sullivan M, Jarosz JM, Martin RJ, et al. MRI hyperintensities of the temporal lobe and external capsule in patients with CADASIL. Neurology 2001;56: 628-634.

38. Dufouil C Chalmers J, Coskun O et al. PROGRESS MRI Substudy Investigators. Effects of blood pressure lowering on cerebral white matter hyperintensities in patients with stroke: the PROGRESS (Perindopril Protection Against Recurrent Stroke Study) Magnetic Resonance Imaging Substudy. Circulation 2005: 112: 1644-1650.

39. Saleh C. Carotid artery intima media thickness: a predictor of cognitive impairment? Front Biosci 2010; 2: 980-990.

40. Birns J, Kalra L. Cognitive function and hypertension. J Hum Hypertens 2009; 23: 86-96.

41. Paran E, Anson O, Reuveni $\mathrm{H}$. Blood pressure and cognitive functioning among independent elderly. Am J Hypertens 2003; 16: 818-826.

42. Nazzaro P, Schirosi G, Mezzapesa D et al. Effect of clustering of metabolic syndrome factors on capillary and cerebrovascular impairment. Eur J Intern Med 2013; 24: 183-188

43. Beason-Held LL, Moghekar A, Zonderman AB et al. Longitudinal changes in cerebral blood flow in the older hypertensive brain. Stroke 2007; 38:1766-1773.

44. Efimova IY, Efimova NY, Triss SV et al. Brain perfusion and cognitive function changes in hypertensive patients. Hypertens Res 2008; 31: 673-678.

45. Kuller LH, Lopez OL, Newman A et al. Risk factors for dementia in the cardiovascular health cognition study. Neuroepidemiology. 2003; 22: 13-22

46. Igase M, Kohara K, Miki T. The association between hypertension and dementia in the elderly. Int J Hypertens 2012 ; doi 10.1155/2012/320648

47. Hajjar I, Hart M, Chen YL et al. Effect of antihypertensive therapy on cognitive function in early executive cognitive impairment: a double blind randomized clinical trial. Arch Intern Med 2012; 172: 442-444. 